\title{
Treatment of Rockwood Type III Acromioclavicular Joint Dislocation
}

\author{
Seong-Hun Kim, Kyoung-Hwan Koh ${ }^{1 凶}$ \\ Department of Orthopedic Surgery, National Health Insurance Service Ilsan Hospital, 'Department of Orthopaedic Surgery, Inje University Ilsan Paik Hospital, \\ Goyang, Korea
}

\begin{abstract}
While non-operative treatment with structured rehabilitation tends to be the strategy of choice in the management of Rockwood type III acromioclavicular joint injury, some advocate surgical treatment to prevent persistent pain, disability, and prominence of the distal clavicle. There is no clear consensus regarding when the surgical treatment should be indicated, and successful clinical outcomes have been reported for non-operative treatment in more than $80 \%$ of type III acromioclavicular joint injuries. Furthermore, there is no gold standard procedure for operative treatment of type III acromioclavicular joint injury, and more than 60 different procedures have been used for this purpose in clinical practice. Among these surgical techniques, recently introduced arthroscopic-assisted procedures involving a coracoclavicular suspension device are minimally invasive and have been shown to achieve successful coracoclavicular reconstruction in $80 \%$ of patients with failed conservative treatment. Taken together, currently available data indicate that successful treatment can be expected with initial conservative treatment in more than $96 \%$ of type III acromioclavicular injuries, whereas minimally invasive surgical treatments can be considered for unstable type IIIB injuries, especially in young and active patients. Further studies are needed to clarify the optimal treatment approach in patients with higher functional needs, especially in high-level athletes.
\end{abstract}

(Clin Shoulder Elbow 2018;21(1):48-55)

Key Words: Acromioclavicular joint; Dislocation; Treatments; Shoulder

\section{Introduction}

Acromioclavicular (AC) joint dislocations are caused by disruption of the AC ligament, coracoclavicular (CC) ligaments, and deltopectoral fascia, resulting in a radiographically widened $\mathrm{AC}$ joint. Such injuries are not uncommonly found in orthopedic practice, accounting for $9 \%$ to $12 \%$ of shoulder injuries. ${ }^{1,2)} \mathrm{AC}$ joint dislocations are classified according to severity, ranging from mild sprains to complete disruption of the AC and CC ligaments. The Rockwood classification is a six-level system widely used to grade $\mathrm{AC}$ injuries, taking into account the integrity of the $\mathrm{AC}$ and CC ligaments, degree of clavicular attachment of the deltoid and trapezius muscles, and direction of dislocation of the clavicle." In general, type I and type II injuries undergo non-operative treatment that involves early range-of-motion and strengthening exercise after short-term immobilization with a sling. On the other hand, type IV, V, and VI injuries are accompanied by significant displacement, with complete rupture of the ligaments, and are thus typically indicated for surgical treatment, as conservative treatment may result in residual pain, weakness, and discomfort in the shoulder and arm. ${ }^{4,5)}$

In Rockwood type III AC joint dislocation, the clavicle is elevated above the superior border of the acromion but the CC distance is less than twice the normal value (25\% to $100 \%$ of the contralateral CC distance) (Fig. 1). ${ }^{6}$ Such injuries are usually treated non-operatively, and this recommendation has been supported over the years by data from many studies. However, non-operative treatment does not achieve active reduction of the dislocation, and the clavicle remains in a prominent position in most patients. Furthermore, long-term studies have reported that non-operative treatment can lead to chronic pain, instability, and degeneration of the AC joint. ${ }^{7,8)}$ Thus, some advocate

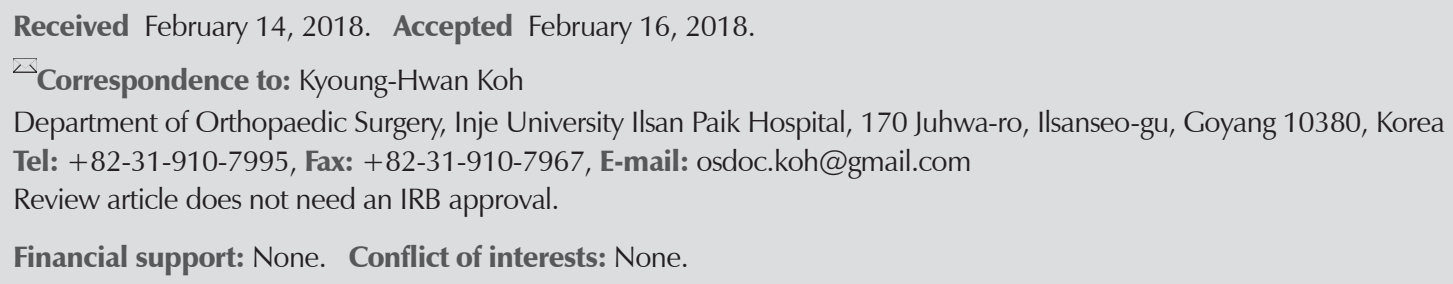




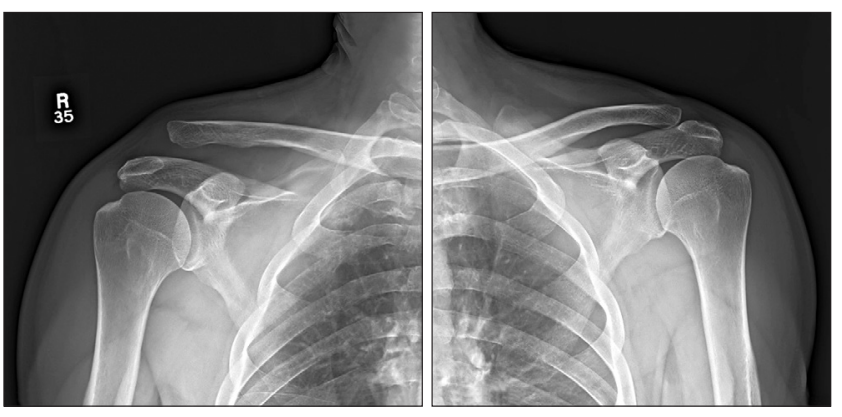

Fig. 1. Representative radiographs revealing type III acromioclavicular joint injury in which the clavicle is elevated above the superior border of the acromion but the coracoclavicular (CC) distance is less than twice the normal value ( $25 \%$ to $100 \%$ of the contralateral CC distance).

surgical treatment of type III AC joint injury in young, physically active adults to maintain good joint power. Shoulder load is typically high in overhead athletes and manual laborers, prompting some surgeons to consider acute surgical treatment in these patients. Several studies have attempted to determine whether conservative or surgical treatment is better for managing type III AC joint dislocation, ${ }^{9-11)}$ but no clear consensus has been established regarding the optimal management of this type of injury, especially in the absence of randomized controlled trials. Thus, there is ongoing debate regarding the optimal treatment strategy in the management of type III AC joint dislocation. Furthermore, there is no gold standard procedure for the operative treatment of the type III injury, as more than 60 different procedures have been described as suitable, which is indicative of the challenges associated with the management of such injuries. ${ }^{12)}$

The present review summarizes the currently available data regarding the management of $\mathrm{AC}$ joint injuries, highlighting the uncertainty surrounding the optimal treatment strategy for type III injuries. The debate shall focus on three key aspects, namely: non-operative versus operative treatment; specific challenges in the treatment of high-level athletes; and overview of operative techniques.

\section{Non-Operative Treatment}

Non-surgical treatment tends to be the treatment of choice in Rockwood type III AC joint injury, based on the findings of several reports. The main goals of non-surgical treatment are relieving pain, promoting the recovery of range of motion, and restoring muscle strength.

Non-operative treatment usually consists of initial sling immobilization and early range-of-motion exercise and rehabilitation programs once pain is alleviated. Special braces such as the Kenny-Howard sling have been reported to provide no substantial benefit over regular slings in terms of long-term clinical outcomes and risk of skin breakdown. ${ }^{13)}$ The rehabilitation program consists of range-of-motion exercise and a combina- tion of isometric and isotonic exercises. Closed-chain exercises should be performed in order to separate scapular movement into individual motions. This approach can also be more tolerable for the patient, because it eliminates the weight of the arm, which decreases joint reactive forces. ${ }^{7)}$ Overall activity should be limited during the period of discomfort, which typically lasts about 6 months.

Non-operative treatment of AC joint dislocation does not restore normal anatomy. Consequently, the deformity persists and there is a potential for residual pain, instability, weakness, and arthritis in the AC joint due to persistent dislocation. Mikek ${ }^{14)}$ evaluated long-term shoulder function after conservative treatment in Rockwood type I and II AC joint injury and found that, at an average follow-up of 10.2 years, more than half of the patients (12 of 23 patients) reported residual AC joint symptoms and unfavorable clinical outcomes compared to the state of the uninjured shoulder. Schlegel et al. ${ }^{15)}$ evaluated 25 patients with acute, untreated type III AC joint dislocation and reported that, at the 3-year follow-up, the injured extremity was, on average, $17 \%$ weaker, as revealed by the strength test. Taft et al. ${ }^{16)}$ reported AC joint arthritis and residual deformity in 37\% and 96\% of patients, respectively, following non-operative treatment of type III AC joint dislocation.

\section{Operative Treatment}

While surgical treatment is typically considered for managing AC joint injuries of type IV, V, and $\mathrm{VI}$, the indication for surgery in type III injuries remains controversial. Operative treatment of type III AC joint dislocation is recommended in patients with failure of non-operative treatment, who typically have persistent pain, scapular dysfunction, and joint weakness. Primary surgical treatment is usually considered for the treatment of type III AC joint dislocation in throwing athletes or manual laborers. Several factors can influence the decision regarding the indication for surgical treatment, ${ }^{17,18)}$ with pre-injury functional demand, current functional deficit, pain, and patient preference for surgery representing the most influential factors; age, sex, and hand dominance generally have small impact on whether or not surgery is offered. ${ }^{19)}$

The Upper Extremity Committee of the International Society of Arthroscopy, Knee Surgery and Orthopedic Sports Medicine recently proposed a sub-classification of the original Rockwood type III dislocation into stable (type IIIA) and unstable (type IIIB) variants. The unstable variant (type IIIB) is characterized by overriding of the distal part of the clavicle on anteroposterior radiograph with cross-arm adduction view (Fig. 2). Patients with type IIIB dislocation have substantial scapular dyskinesia that does not respond to physical therapy and may benefit from early operative fixation. ${ }^{20)}$

Operative treatment attempts to restore normal anatomy but 


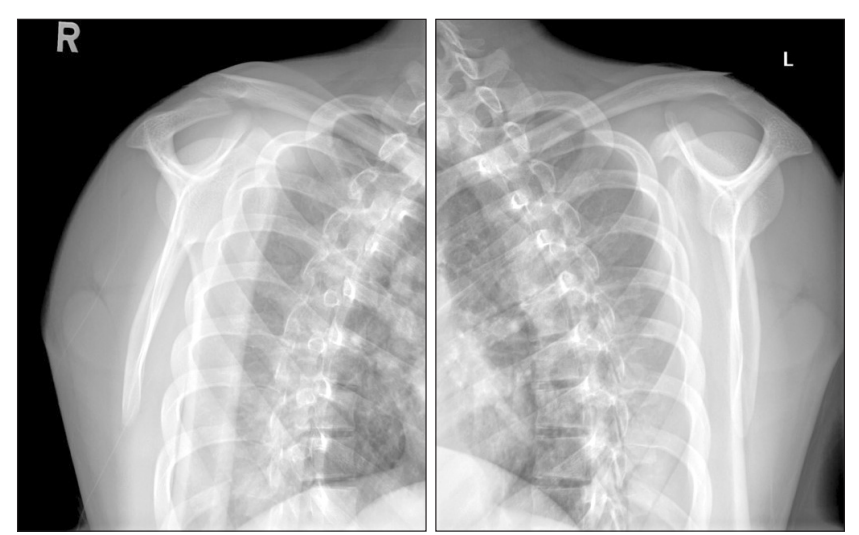

Fig. 2. Supraspinatus outlet view revealing overriding of the distal clavicle. According to the Upper Extremity Committee of the International Society of Arthroscopy, Knee Surgery and Orthopedic Sports Medicine, unstable type IIIB acromioclavicular joint injury is characterized by overriding of the distal part of the clavicle on anteroposterior radiograph with cross-arm adduction view.

can be associated with a variety of complications. A common surgical complication is loss of reduction, which can occur due to loss of fixation, fracture of the coracoid or clavicle, biologic failure as a result of gradual stretching of the graft construct, failure of the hardware device, erosions through the clavicle or coracoid in fractures augmented with non-absorbable tape or suture, and migration of transfixing pins or wires. Other complications associated with surgery include tunnel osteolysis, infection, ossification of the CC or AC ligament, and osteoarthritis of the $\mathrm{AC}$ joint. ${ }^{1,2,21)}$

\section{Non-Operative versus Operative Treatment}

The optimal treatment of type III AC injuries remains controversial, and this topic has been extensively debated in the literature, with many reports providing data, but none from highquality, randomized studies. In a prospective study by Bannister et al. ${ }^{22)} 60$ patients with acute AC dislocation were randomly allocated to receive treatment with a sling or fixation with a CC screw. At the 4-year follow-up, $85 \%$ of surgically treated patients and $100 \%$ of non-operatively treated patients had satisfactory outcome. The authors reported that patients with initial displacement below $2 \mathrm{~cm}$ performed better with non-operative intervention, while those with greater displacement were more likely to benefit from surgery. Phillips et al. ${ }^{23)}$ performed a metaanalysis of 24 studies, including a total of 1,172 patients, and found satisfactory outcomes after both operative $(88 \%)$ and nonoperative (87\%) treatment. In a critical analysis review, Virk et al. ${ }^{24)}$ reported similar results. Of the 14 studies comparing operative with non-operative treatment, three were prospective (level of evidence: II), whereas the remaining eleven were retrospective (level of evidence: III). A cumulative total of 706 patients were included in these studies. Favorable clinical outcome (de- fined as good or better) was reported in $88 \%$ of the operatively managed patients and in $85.5 \%$ of the non-operatively managed patients. Anatomic reduction of the joint was achieved in 59\% of the operatively managed patients and in only $14.7 \%$ of the non-operatively managed patients.

Overall, most studies indicate that there is no difference in clinical outcomes between operative and non-operative treatment of type III AC joint dislocation. However, further studies are needed, especially high-quality, randomized, controlled trials.

\section{Debate Regarding the Optimal Strategy in High-Level Athletes}

There is a question as to whether the outcomes of surgical or non-surgical treatment for type III AC joint dislocation, as described above, can be maintained in high-level athletes, who must maintain high levels of physical activity. A national survey from the United Kingdom ${ }^{19)}$ revealed that patients with high preinjury functional demand are typically indicated for primary surgical treatment. However, data from studies evaluating treatment outcomes in athletes support both operative and non-operative strategies.

Tibone et al. ${ }^{25)}$ evaluated 20 athletes treated without surgery, and concluded that conservative treatment achieves restoration of joint strength in the injured shoulder to a level comparable to that of the uninjured shoulder, as revealed by strength testing with a Cybex II dynamometer. These findings suggest that conservative treatment results in minimal or no functional deficit, and the authors recommend that type III AC dislocations be treated non-operatively even in athletes. Glick et al. ${ }^{26)}$ retrospectively evaluated 34 male patients with complete AC joint dislocation, and reported similar findings for a mean follow-up of about 3 years. In their study, most patients were athletes (19/34 were professional football players, and 9/34 were competitive or recreational athletes). The patients were managed with an aggressive rehabilitation protocol, and most of the competitive athletes returned to playing their sport within four weeks. At the time of evaluation, all professional football players had full range of shoulder motion and reported feeling no pain in the affected AC joint. A study by Gurd ${ }^{27}$ also supported the idea that rehabilitation was the primary factor resulting in a successful outcome. McFarland et al. ${ }^{18)}$ evaluated the outcome of operative and nonoperative treatment in major league baseball players with type III AC joint dislocation in the throwing arm. They reported that $80 \%$ of the patients treated non-operatively regained normal function and achieved complete relief of pain, while $90 \%$ had normal range of motion after treatment; of those treated operatively, 92\% regained normal function, achieved complete relief of pain, and had normal range of motion after surgery.

On the other hand, Galpin et al. ${ }^{28}$ found that $33 \%$ of throwing athletes treated non-operatively did not recover full throwing 
ability. Similarly, Wojtys and Nelson ${ }^{29)}$ reported incomplete recovery in $42 \%$ of throwers included in their study. Therefore, the optimal treatment strategy for type III AC dislocation in athletes remains controversial. Further study is warranted in this area, primarily regarding throwing athletes.

\section{Overview of Operative Techniques}

There is a wide range of options for the operative treatment of AC joint injuries. Many different surgical procedures have been proposed, and there is no clear agreement regarding the superiority of one technique over another. There are two broad categories of surgical treatment methods used in the treatment of AC joint injuries: one category includes methods that seek to provide fixation across the AC joint or $\mathrm{CC}$ joint, while the other includes methods that seek to augment or reconstruct the AC or CC ligaments. Ligament reconstruction can be further differentiated into anatomic and non-anatomic reconstruction.

\section{Fixation of the Acromioclavicular Joint}

AC joint fixation allows time for the native AC and CC ligament to heal in place by reducing the $\mathrm{AC}$ joint and maintaining reduced AC joint. The technique involves closed or open reduction of the joint, followed by repair of the AC ligament and damaged deltoid and trapezius attachments, with stabilization conferred by direct fixation across the joint. Eskola et al. ${ }^{30}$ studied 86 patients with complete AC joint dislocation treated with one of three different methods including transfixation with two smooth Kirschner wires, with two threaded wires, and with one cortical screw. At the one-year follow-up, favorable results were obtained in 82 patients. However, due to complications such as pin migration, this technique has been largely abandoned."

Hook-plate fixation is another method of primary fixation across the AC joint (Fig. 3). The plate was first introduced for this purpose in 1976 by Balser, ${ }^{31)}$ and was later adopted widely in clinical practice across Europe. In this method, the hook portion of the plate is positioned beneath the acromion, and the plate is then fixed to the clavicle with screws, maintaining adequate reduction of the AC joint. The technique can be used to treat acute injuries and may be combined with ligament reconstruction for chronic injuries, with good short-term outcomes. Kienast et al. ${ }^{32)}$ used AC hook-plate fixation to treat 225 patients with Rockwood type III-V AC joint dislocation, and reported excellent or good outcomes in $89 \%$ of patients, but the overall rate of complications was relatively high $(\mathbf{1 0 . 6 \% )})$. It is not uncommon for hook-plate fixation to result in complications, including persistent erosion of the acromial undersurface, fracture of the clavicle medial to the plate, dislodgement of the hook, redislocation, and subluxation. ${ }^{33,34)}$ The main disadvantage of this surgical procedure is the need for a second surgery for hardware removal. If the metal plate is not removed, widening of the hook

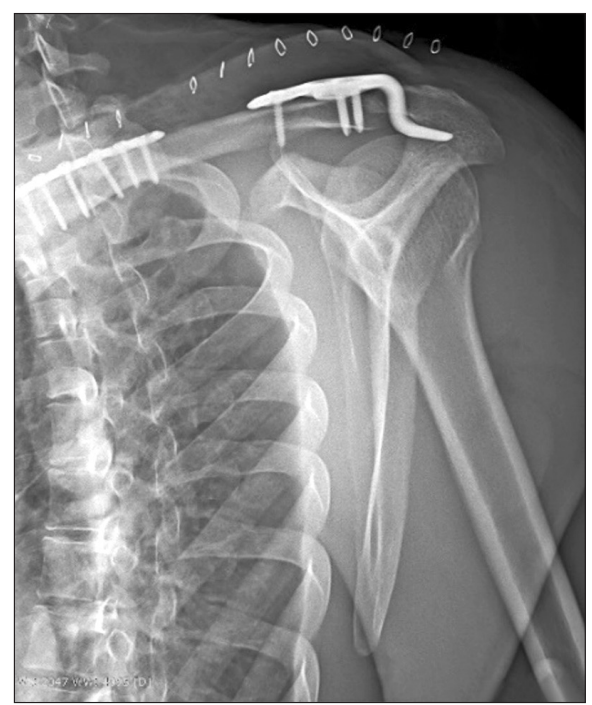

Fig. 3. Plain radiograph revealing acromioclavicular fixation using a Hook plate.

hole in the acromion usually develops, secondary to normal motion of the AC joint. ${ }^{34)}$

\section{Fixation of the Coracoclavicular Joint}

In 1941, Bosworth ${ }^{35}$ introduced a fixation technique involving the placement of a screw between the clavicle and the coracoid. Typically, a $6.5-\mathrm{mm}$ partially threaded cancellous screw is used. Most surgeons favor open screw insertion, as percutaneous techniques have been reported to be associated with a high rate of technical failure (32\%). ${ }^{36)}$ According to Rockwood et al., ${ }^{37)}$ five types of motion between the coracoid and the clavicle can lead to fatigue or failure of the implant over time. Because of the high rate of hardware migration and screw breakage over time, re-operation is usually required between 8 and 12 weeks after the index surgery. ${ }^{33)}$ A CC sling can be created using various sutures and implants such as Mersilene tape, suture anchors, a small metallic flip button, and non-absorbable sutures. ${ }^{7)}$

In the management of AC joint injuries, arthroscopy-assisted procedures provide several advantages over open procedures. The main advantages include reduced morbidity associated with minimally invasive approaches, the possibility to diagnose and treat concomitant intra-articular injuries, and the possibility of direct visualization of the inferior aspect of the base of the coracoid. Arthroscopic-assisted procedures with a CC suspension device aim to reduce the $\mathrm{CC}$ distance, allowing time for healing of the torn AC and CC ligaments. A prosthetic CC suspension device such as the TightRope device (Arthrex, Naples, FL, USA) can be implanted (Fig. 4), with titanium buttons placed on top of the clavicle and under the coracoid, and connected with a continuous loop of no. 5 FiberWire suture (Arthrex). Good clinical outcomes have been reported for the use of one suspension device placed at the isometric point of the CC ligaments, but the risk of secondary subluxation remains of concern. ${ }^{38}$ However, using a 

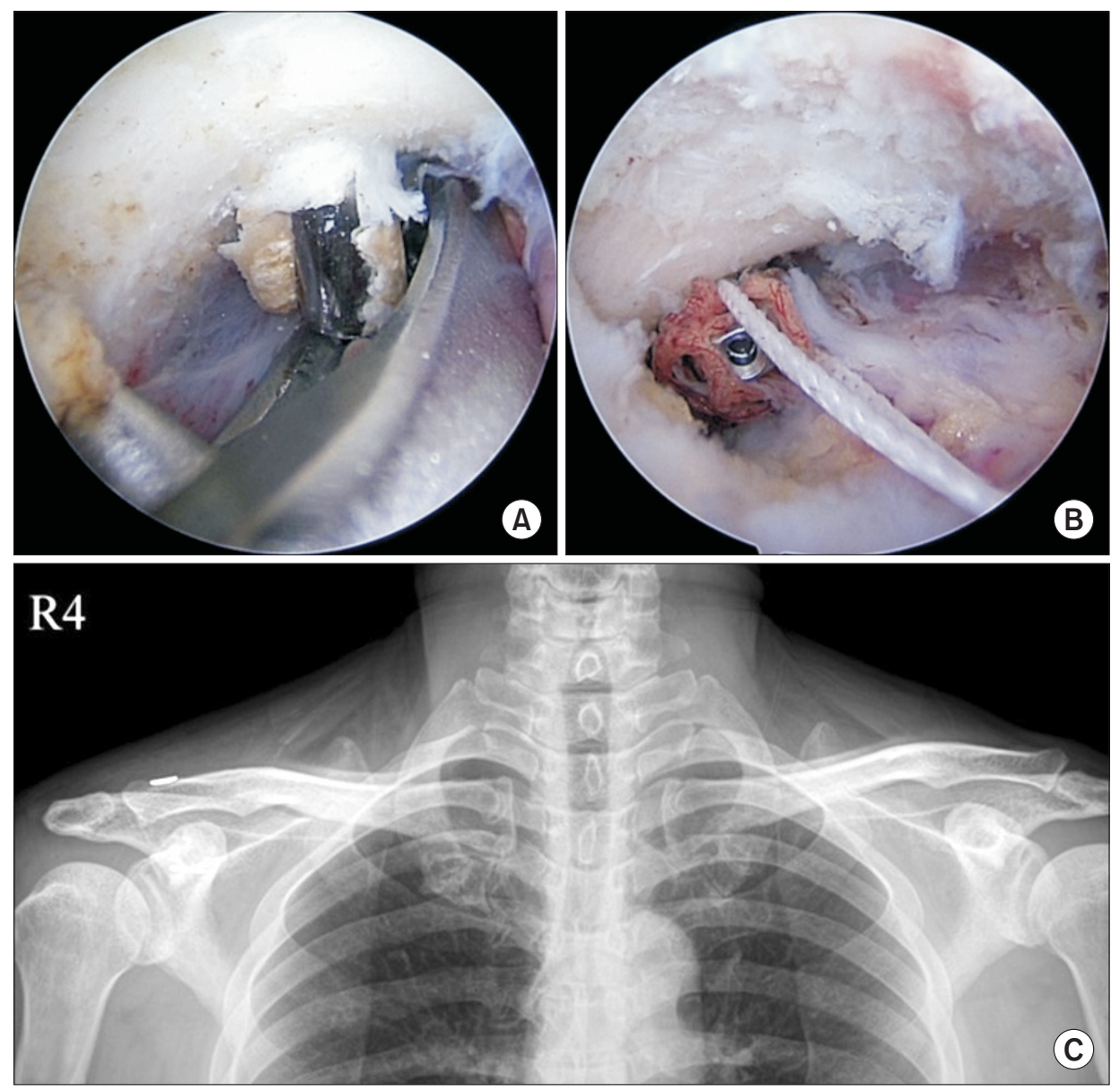

Fig. 4. Arthroscopic-assisted procedures with a coracoclavicular suspension device. (A, B) Formation of the coracoid tunnel using a targeting device, and insertion of a suspension device. (C) Postoperative anteroposterior radiograph revealing slightly over-reduced coracoclavicular interval and acromiohumeral joint.

single isometric CC suspension device does not reproduce the biomechanics of the CC ligaments, and the fixation seems to be insufficient to maintain the reduction of the AC joint. In a biomechanical study by Walz et al., ${ }^{39)}$ reconstruction of the conoid and trapezoid ligaments by means of two TightRope devices led to favorable outcomes in vitro, providing equal or even higher forces than those noted in native CC ligaments. Scheibel et al. ${ }^{40)}$ used an arthroscopic-assisted technique involving two TightRope devices to treat 28 patients with acute AC joint dislocation, and reported good to excellent early clinical outcomes despite partial, recurrent, vertical and horizontal instability in the AC joint. Later on, Venjakob et al. ${ }^{41)}$ reported satisfactory clinical outcomes at a mean follow-up of 58 months in 23 patients with acute unstable AC joint injury treated with arthroscopically assisted reduction and anatomic fixation using two suture-button devices. According to the biomechanical and clinical evidence currently available, synthetic anatomic reconstruction of both the conoid and trapezoid ligaments using two suspension devices placed in the native origins of the torn structures can be considered a feasible and reliable treatment strategy.

\section{Ligament Reconstruction}

Ligaments that achieve complete healing in place in the acute period can have the same tensile strength as undamaged ligaments. If the ligaments do not heal completely, fixation and repair failure can occur as a result of cyclical loading of the joint. Another approach is to reconstruct the CC ligaments with transfer of local tissues or with free tendon grafts. There are many procedures in use today for reconstruction of the AC joint, and these can be differentiated based on whether ligament reconstruction is anatomic or non-anatomic.

Weaver and Dunn ${ }^{42)}$ first described the traditional nonanatomic technique, which involves transferring the proximal portion of the coracoacromial ligament to the distal end of the clavicle. The modified Weaver-Dunn procedure has been reported to provide good to excellent outcomes in $75 \%$ of patients. ${ }^{42)}$ However, the initial strength of the coracoacromial ligament after transfer is only $25 \%$ of the normal value, and stability in the anteroposterior plane is not restored. ${ }^{43)}$ Up to $30 \%$ of recurrent subluxation has been reported to be due to the lack of initial strength. ${ }^{44)}$

In current clinical practice, ligament reconstruction involves the use of allogenic or autogenous grafts to reconstruct the AC and CC ligaments. Anatomic reconstruction is known to provide favorable outcomes in patients with delayed surgery or failed conservative management. Several biomechanical studies in- 
volving cadaveric specimens have demonstrated that anatomic CC ligament reconstruction provides superior biomechanical properties than those obtained using conventional non-anatomic ligament reconstruction (i.e., the Weaver-Dunn procedure). ${ }^{45-47}$ Furthermore, AC ligament reconstruction in combination with anatomic CC ligament reconstruction provides superior rotational and translational stability to the AC joint complex. ${ }^{48)}$

In the clinical study by Nicholas et al., ${ }^{49}$ satisfactory outcomes and no loss of reduction of the AC joint were noted in 8 of 9 patients with CC ligament reconstruction using semitendinosus tendon allografts. In a recent study, Garofalo et al. ${ }^{50)}$ found that 93\% of patients could return to their pre-injury level of work and sports activities following CC ligament reconstruction in combination with $\mathrm{AC}$ ligament reconstruction.

In a comparative study, Tauber et al. ${ }^{511}$ prospectively evaluated 24 patients with chronic AC joint dislocation managed using the Weaver-Dunn procedure or anatomic reconstruction of CC ligaments with autogenous semitendinosus tendon grafts. The authors concluded that, compared to patients treated with the modified Weaver-Dunn procedure, those treated with anatomic CC ligament reconstruction had significantly superior clinical and radiologic outcomes.

An important point to note is that the learning curve for anatomic ligament reconstruction is steep. The coracoid and clavicle are thin bones, prone to fracture, and extensive manipulation during ligament reconstruction carries the risk of complications. Cook et al. ${ }^{52)}$ reported a $28 \%$ failure rate at an average of 7.4 weeks following CC ligament reconstruction, due to malposition of the clavicular bone tunnel. In a study by Milewski et al., ${ }^{53)}$ coracoid fractures $(7 \%)$ and clavicle fractures $(11 \%)$ were noted in a series of 27 patients who underwent CC ligament reconstruction AC joint dislocation. Although promising, the anatomic reconstruction of CC ligaments should be undertaken with caution, and further studies are necessary to clarify the long-term benefits of this procedure.

\section{Conclusion}

AC joint dislocation is a relatively common injury in the general population. There is a general consensus that type I and II injuries should be treated non-operatively, whereas surgical treatment may be considered for type IV, V, and VI injuries. However, there is no consensus regarding the optimal treatment strategy for type III injuries. In the average patient, non-operative treatment tends to be the preferred strategy provided that there is no persistent pain, instability, or functional limitation after conservative treatment. Many studies have reported good clinical outcomes following non-operative treatment in more than $80 \%$ of patients with acute type III AC joint injury, suggesting that surgical treatment may provide no added benefit. Furthermore, if conservative treatment fails, favorable clinical outcomes can still be obtained with anatomic CC ligament reconstruction in over $80 \%$ of patients with chronic type III AC joint injury. Taken together, these data suggest that successful treatment outcomes can be expected in more than $96 \%$ of patients with type III AC joint dislocation with initial conservative treatment. However, further studies are needed to determine the optimal treatment strategy in patients with high preoperative functional needs, especially in high-level athletes. Additionally, early operative treatment in the acute phase may be considered in patients with unstable type IIIB AC joint injury with therapy-resistant scapular dysfunction and overriding of the clavicle in the cross-body adduction view. Surgical methods have advanced a great deal over the past 30 years, and various surgical procedures have been reported to be suitable for treating AC joint injuries. However, no gold standard procedure has been established. In general, AC joint fixation methods using metal hardware are less advantageous because of higher complication rates and the need for a second surgery for hardware removal. Thus, non-operative treatment is recommended as the first-line strategy in patients with type III AC joint dislocation unless they have unstable type IIIB injury, in which case surgical treatment might be considered first, preferably involving open or arthroscopic-assisted CC suspension without metal hardware, to reduce the risk of complications and avoid a second surgery. In patients with persistent symptoms due to failure of conservative treatment, anatomic CC ligament reconstruction in the chronic stage is likely to provide successful outcomes.

\section{References}

1. Fraser-Moodie JA, Shortt NL, Robinson CM. Injuries to the acromioclavicular joint. J Bone Joint Surg Br. 2008;90(6):697707.

2. Mazzocca AD, Arciero RA, Bicos J. Evaluation and treatment of acromioclavicular joint injuries. Am J Sports Med. 2007;35(2):316-29.

3. Rockwood CA, Green DP. Fractures in adults. 2nd ed. Philadelphia: Lippincott; 1984.

4. Beitzel K, Cote MP, Apostolakos J, et al. Current concepts in the treatment of acromioclavicular joint dislocations. Arthroscopy. 2013;29(2):387-97.

5. Mulier T, Stuyck J, Fabry G. Conservative treatment of acromioclavicular dislocation. Evaluation of functional and radiological results after six years follow-up. Acta Orthop Belg. 1993;59(3):255-62.

6. Rockwood CA, Green DP. Rockwood and Green's fractures in adults. 4th ed. Philadelphia: Lippincott-Raven; 1996.

7. Kim S, Blank A, Strauss E. Management of type 3 acromioclavicular joint dislocations: current controversies. Bull Hosp Jt Dis (2013). 2014;72(1):53-60.

8. Calvo E, López-Franco M, Arribas IM. Clinical and radio- 
logic outcomes of surgical and conservative treatment of type III acromioclavicular joint injury. J Shoulder Elbow Surg. 2006;15(3):300-5.

9. Smith TO, Chester R, Pearse EO, Hing CB. Operative versus non-operative management following Rockwood grade III acromioclavicular separation: a meta-analysis of the current evidence base. J Orthop Traumatol. 2011;12(1):19-27.

10. Spencer EE Jr. Treatment of grade III acromioclavicular joint injuries: a systematic review. Clin Orthop Relat Res. 2007;455: 38-44.

11. Tamaoki MJ, Belloti JC, Lenza M, Matsumoto MH, Gomes Dos Santos JB, Faloppa F. Surgical versus conservative interventions for treating acromioclavicular dislocation of the shoulder in adults. Cochrane Database Syst Rev. 2010;(8):CD007429.

12. Stucken $C$, Cohen SB. Management of acromioclavicular joint injuries. Orthop Clin North Am. 2015;46(1):57-66.

13. Allman FL Jr. Fractures and ligamentous injuries of the clavicle and its articulation. J Bone Joint Surg Am. 1967;49(4):774-84.

14. Mikek M. Long-term shoulder function after type I and II acromioclavicular joint disruption. Am J Sports Med. 2008; 36(11):2147-50.

15. Schlegel TF, Burks RT, Marcus RL, Dunn HK. A prospective evaluation of untreated acute grade III acromioclavicular separations. Am J Sports Med. 2001;29(6):699-703.

16. Taft TN, Wilson FC, Oglesby JW. Dislocation of the acromioclavicular joint. An end-result study. J Bone Joint Surg Am. 1987;69(7):1045-51.

17. Cardone D, Brown JN, Roberts SN, Saies AD, Hayes MG. Grade III acromioclavicular joint injury in Australian rules football. J Sci Med Sport. 2002;5(2):143-8.

18. McFarland EG, Blivin SJ, Doehring CB, Curl LA, Silberstein C. Treatment of grade III acromioclavicular separations in professional throwing athletes: results of a survey. Am J Orthop (Belle Mead NJ). 1997;26(11):771-4.

19. Domos P, Sim F, Dunne M, White A. Current practice in the management of Rockwood type III acromioclavicular joint dislocations-National survey. J Orthop Surg (Hong Kong). 2017;25(2):2309499017717868.

20. Beitzel K, Mazzocca AD, Bak K, et al. ISAKOS upper extremity committee consensus statement on the need for diversification of the Rockwood classification for acromioclavicular joint injuries. Arthroscopy. 2014;30(2):271-8.

21. Simovitch R, Sanders B, Ozbaydar M, Lavery K, Warner JJ. Acromioclavicular joint injuries: diagnosis and management. J Am Acad Orthop Surg. 2009;17(4):207-19.

22. Bannister GC, Wallace WA, Stableforth PG, Hutson MA. The management of acute acromioclavicular dislocation. A randomised prospective controlled trial. J Bone Joint Surg Br. 1989;71(5):848-50.

23. Phillips AM, Smart C, Groom AF. Acromioclavicular dislocation. Conservative or surgical therapy. Clin Orthop Relat Res.
1998;(353):10-7.

24. Virk MS, Apostolakos J, Cote MP, Baker B, Beitzel K, Mazzocca AD. Operative and nonoperative treatment of acromioclavicular dislocation: a critical analysis review. JBJS Rev. 2015;3(10):01874474-201510000-00006. doi: 10.2106/JBJS. RVW.N.00092.

25. Tibone J, Sellers R, Tonino P. Strength testing after thirddegree acromioclavicular dislocations. Am J Sports Med. 1992;20(3):328-31.

26. Glick JM, Milburn LJ, Haggerty JF, Nishimoto D. Dislocated acromioclavicular joint: follow-up study of 35 unreduced acromioclavicular dislocations. Am J Sports Med. 1977;5(6):26470.

27. Gurd FB. The treatment of complete dislocation of the outer end of the clavicle: an hitherto undescribed operation. Ann Surg. 1941;113(6):1094-8.

28. Galpin RD, Hawkins RJ, Grainger RW. A comparative analysis of operative versus nonoperative treatment of grade III acromioclavicular separations. Clin Orthop Relat Res. 1985;(193): 150-5.

29. Wojtys EM, Nelson G. Conservative treatment of Grade III acromioclavicular dislocations. Clin Orthop Relat Res. $1991 ;(268): 112-9$.

30. Eskola A, Vainionpää S, Korkala O, Rokkanen P. Acute complete acromioclavicular dislocation. A prospective randomized trial of fixation with smooth or threaded Kirschner wires or cortical screw. Ann Chir Gynaecol. 1987;76(6):323-6.

31. Balser D. Eine neue moethode zur operative behandlung der akromioklavikularen luxation. Chir Prax. 1976;24:275.

32. Kienast B, Thietje R, Queitsch C, Gille J, Schulz AP, Meiners J. Mid-term results after operative treatment of rockwood grade III-V acromioclavicular joint dislocations with an AC-hookplate. Eur J Med Res. 2011;16(2):52-6.

33. Kwon YW, lannotti JP. Operative treatment of acromioclavicular joint injuries and results. Clin Sports Med. 2003;22(2):291300 , vi.

34. Sim E, Schwarz N, Höcker K, Berzlanovich A. Repair of complete acromioclavicular separations using the acromioclavicular-hook plate. Clin Orthop Relat Res. 1995;(314):134-42.

35. Bosworth BM. Acromioclavicular separation: New method of repair. Surg Gynecol Obset 1941;73:866-71.

36. Tsou PM. Percutaneous cannulated screw coracoclavicular fixation for acute acromioclavicular dislocations. Clin Orthop Relat Res. 1989;(243):112-21.

37. Rockwood CA, Green DP, Bucholz RW. Rockwood and Green's fractures in adults. 6th ed. Philadelphia: Lippincott Williams \& Wilkins; 2006.

38. Shin SJ, Kim NK. Complications after arthroscopic coracoclavicular reconstruction using a single adjustable-loop-length suspensory fixation device in acute acromioclavicular joint dislocation. Arthroscopy. 2015;31(5):816-24. 
39. Walz L, Salzmann GM, Fabbro T, Eichhorn S, Imhoff AB. The anatomic reconstruction of acromioclavicular joint dislocations using 2 TightRope devices: a biomechanical study. Am J Sports Med. 2008;36(12):2398-406.

40. Scheibel M, Dröschel S, Gerhardt C, Kraus N. Arthroscopically assisted stabilization of acute high-grade acromioclavicular joint separations. Am J Sports Med. 2011;39(7):1507-16.

41. Venjakob AJ, Salzmann GM, Gabel F, et al. Arthroscopically assisted 2-bundle anatomic reduction of acute acromioclavicular joint separations: 58-month findings. Am J Sports Med. 2013;41(3):615-21.

42. Weaver JK, Dunn HK. Treatment of acromioclavicular injuries, especially complete acromioclavicular separation. J Bone Joint Surg Am. 1972;54(6):1187-94.

43. Lee SJ, Nicholas SJ, Akizuki KH, McHugh MP, Kremenic IJ, Ben-Avi S. Reconstruction of the coracoclavicular ligaments with tendon grafts: a comparative biomechanical study. Am J Sports Med. 2003;31(5):648-55.

44. Weinstein DM, McCann PD, Mcllveen SJ, Flatow EL, Bigliani LU. Surgical treatment of complete acromioclavicular dislocations. Am J Sports Med. 1995;23(3):324-31.

45. Grutter PW, Petersen SA. Anatomical acromioclavicular ligament reconstruction: a biomechanical comparison of reconstructive techniques of the acromioclavicular joint. Am J Sports Med. 2005;33(11):1723-8.

46. Mazzocca AD, Santangelo SA, Johnson ST, Rios CG, Dumonski ML, Arciero RA. A biomechanical evaluation of an anatomical coracoclavicular ligament reconstruction. Am J Sports Med. 2006;34(2):236-46.
47. Thomas K, Litsky A, Jones G, Bishop JY. Biomechanical comparison of coracoclavicular reconstructive techniques. Am J Sports Med. 2011;39(4):804-10.

48. Beitzel K, Obopilwe E, Apostolakos J, et al. Rotational and translational stability of different methods for direct acromioclavicular ligament repair in anatomic acromioclavicular joint reconstruction. Am J Sports Med. 2014;42(9):2141-8.

49. Nicholas SJ, Lee SJ, Mullaney MJ, Tyler TF, McHugh MP. Clinical outcomes of coracoclavicular ligament reconstructions using tendon grafts. Am J Sports Med. 2007;35(11):1912-7.

50. Garofalo R, Ceccarelli E, Castagna A, et al. Open capsular and ligament reconstruction with semitendinosus hamstring autograft successfully controls superior and posterior translation for type V acromioclavicular joint dislocation. Knee Surg Sports Traumatol Arthrosc. 2017;25(7):1989-94.

51. Tauber M, Gordon K, Koller H, Fox M, Resch H. Semitendinosus tendon graft versus a modified Weaver-Dunn procedure for acromioclavicular joint reconstruction in chronic cases: a prospective comparative study. Am J Sports Med. 2009;37(1):181-90.

52. Cook JB, Shaha JS, Rowles DJ, Bottoni CR, Shaha SH, Tokish JM. Clavicular bone tunnel malposition leads to early failures in coracoclavicular ligament reconstructions. Am J Sports Med. 2013;41(1):142-8.

53. Milewski MD, Tompkins M, Giugale JM, Carson EW, Miller MD, Diduch DR. Complications related to anatomic reconstruction of the coracoclavicular ligaments. Am J Sports Med. 2012;40(7):1628-34. 